\title{
Déchets municipaux dans le District d'Abidjan en Côte d'Ivoire: sources potentielles de pollution des eaux souterraines
}

\author{
Nagnin SORO ${ }^{1 *}$, Lazéni OUATTARA ${ }^{1}$, Kouassi DONGO ${ }^{1}$, \\ Emmanuel Konan KOUADIO ${ }^{1}$, Ernest Kouassi AHOUSSI ${ }^{1}$, Gbombélé SORO ${ }^{1}$, \\ Marie-Solange OGA, ${ }^{1}$ Issiaka SAVANE ${ }^{2}$ et Jean BIEMI ${ }^{1}$ \\ ${ }^{1}$ Laboratoire des Sciences et Techniques de l'Eau et de l'Environnement, UFR Sciences de la Terre et des \\ Ressources Minières, Université de Cocody, 22 B.P. 582 Abidjan 22, Côte d'Ivoire. \\ ${ }^{2}$ Laboratoire de Géosciences et Environnement, UFR Sciences et Gestion de l'Environnement, Université \\ Abobo-Adjamé 08 B.P. 109 Abidjan 08 Côte d'Ivoire, Tél. (225) 21257336 \\ *Auteur correspondant ; E-mail: soro_nagnin@yahoo.fr; Tél. (225) 22483803
}

\section{RESUME}

La population de la ville d'Abidjan s'est considérablement accrue ces dernières années. Cette croissance s'est accompagnée d'une forte urbanisation et d'une croissance industrielle non contrôlées. A cela s'ajoute une absence de structure de surveillance efficace et surtout un non respect de la réglementation environnementale. La production importante de déchets domestiques, industriels et hospitaliers qui en résulte, est confrontée à une gestion insuffisante et à des problèmes d'infrastructures et de difficultés économiques. Les quantités de déchets générés en 2002, par la ville sont estimées à 1225688,97 tonnes (soit $0,98 \mathrm{~kg} / \mathrm{ha} /$ jour) contre $193186 \mathrm{~m}^{3} / \mathrm{j}$ de volume d'eaux usées domestiques. Le réseau d'assainissement présente un faible taux de couverture de la population (29\%) en raison du dysfonctionnement de la quasi-totalité des ouvrages existant. Cette situation entraîne un rejet journalier dans la nature, hors des systèmes d'égout, de plus de $115912 \mathrm{~m}^{3}$ soit $60 \%$ des eaux usées avec une charge polluante correspondante estimée à plus de $56113 \mathrm{~kg}$ de $\mathrm{DBO}_{5}$. A cela s'ajoute l'accumulation, chaque année, de près de 550000 tonnes de déchets ménagers non ramassés et dont les corollaires sont l'engorgement permanent de toutes les communes d'une quantité importante de déchets ménagers et partant des difficultés d'écoulement des eaux usées et pluviales. Mais plus particulièrement, ce sont les communes de Yopougon, Adjamé, Port-Bouët, Cocody et Koumassi où les taux de ramassage des ordures ménagères sont généralement inférieurs à $50 \%$ de la production locale qui sont les plus touchées. Cette situation a des répercussions sur la qualité des eaux de surface mais aussi celles souterraines dont la protection est de plus en plus menacée par une urbanisation anarchique et par des pompages excessifs aux fins de répondre aux besoins sans cesse croissants de la population. L'évaluation de l'état de pollution de la nappe d'Abidjan a été menée à partir des données de forages des nombreux champs de captage de la ville, sur la période allant de 1995 à 2001. Ceci a permis de relever des teneurs anormalement élevées (au dessus de la norme OMS) en composés azotés $\left(\mathrm{NO}_{3}, \mathrm{NO}_{2}, \mathrm{NH}_{4}\right)$, phosphatés $\left(\mathrm{PO}_{4}\right)$ et en élément métallique $(\mathrm{Al})$. Par ailleurs, les analyses chimiques des eaux souterraines de l'année 2000 issues de 56 forages ont fait l'objet d'une étude statistique. L'utilisation des techniques de cartographie et de l'analyse en composantes principales (ACP) s'est avérée nécessaire dans l'identification de l'origine de cette pollution. Elle serait due, entre autres, aux apports superficiels issus de la décomposition de la matière organique fermentescible mais également aux eaux putrides provenant de la surface du sol et entraînant avec elles des polluants vers la nappe. Cette contamination progressive de la nappe se fait du Sud vers le Nord en direction des nouveaux pôles d'habitation et des nouvelles zones industrielles.

Mots-clés : Déchets solides, eaux usées, composés azotés, composés phosphatés, pollution, Abidjan 


\section{INTRODUCTION}

Dans la plupart des pays en développement (PED) on assiste à un exode massif des populations rurales, vers les grandes villes, en quête d'un meilleur avenir. Ces populations, bien évidemment, viennent s'installer à la périphérie des villes ou dans des quartiers dits précaires difficilement accessibles puisque situés sur des sites à haut risque et interdits de construction. L'accroissement des populations urbaines, l'installation de nombreuses unités industrielles et la naissance d'une agriculture périurbaine ont pour conséquence la production d'une quantité importante de déchets (solides ou liquides) qui sont mal gérés. Cette situation est amplifiée, dans de nombreux cas, par l'absence d'infrastructures adaptées liées à une urbanisation anarchique et mal contrôlée. Ainsi l'un des problèmes majeurs auxquels les responsables communaux des grandes villes africaines doivent constamment faire face est celui de la gestion durable de ces déchets urbains et l'accès, pour les populations, à un système d'assainissement adéquat.

La ville d'Abidjan, une des plus importantes métropoles d'Afrique de l'Ouest, dont la population a triplé en l'espace de deux décennies (INS, 1998) vit au quotidien cette réalité. En effet, la collecte des eaux usées est insuffisante car l'assainissement n'a pas suivi l'extension de la ville et la croissance démographique. Le système d'assainissement présente un faible taux de raccordement aux ménages (29\%) et des dysfonctionnements dus à la dégradation de la quasi-totalité des ouvrages (stations et canalisations) prévalents. Cette situation entraîne le rejet d'importantes quantités d'eaux usées hors des systèmes d'égout.

Par ailleurs, le taux de collecte des ordures ménagères est estimé à $55 \%$ de la production totale estimée à plus d'un million de tonnes (BNETD, 2000). Les déchets non collectés, en grande partie putrescibles se retrouvent dans les ravins, les bas-fonds, les baies lagunaires, les canalisations d'eaux usées et pluviales, les puits abandonnés et même autour des périmètres de protection rapprochés des champs de captage. L'élimination pendant environ 30 ans des déchets urbains à la décharge d'Akouédo, sans mesure de protection de l'environnement, entraîne un écoulement permanent vers les eaux lagunaires du lixiviat qui pourraient rejoindre les eaux souterraines (Groupe Eoule, 2000; Kouamé et al., 2006a in Adjiri, 2008). En effet, des possibilités de circulation verticale des eaux de surface vers les nappes de profondeur existent partout où l'épaisseur des niveaux quaternaires est assez faible. De même, cette possibilité est accentuée lorsque la cuirasse latéritique imperméable coiffant la nappe du Continental Terminal (MioPliocène) est absente, permettant l'interconnexion verticale entre les nappes.

Le rejet continu et permanent d'importantes quantités de déchets liquides et solides dans les milieux naturels favorise la dispersion et la diffusion d'éléments polluants dans les milieux récepteurs. La qualité des eaux de la nappe du Continental Terminal (CT) est de plus en plus menacée par les activités anthropiques. Ainsi, des cas de pollutions ont été signalés dans des études antérieures (Aghui et Biémi, 1984; Jourda, 1987; Kouadio, 1997; Oga, 1998; Ahoussi, 2008) et ceux de fortes minéralisations liées à l'abondance de nitrates ont été observés par Jourda (1987) et Ahoussi (2008). Ceci montre que la nappe du Continental Terminal n'est pas à l'abri de toute pollution. Son ampleur est mal connue et son impact sur les ressources en eaux est pratiquement sous-estimé.

La présente étude vise à évaluer les conséquences de la gestion des déchets urbains sur la qualité des eaux souterraines de la nappe d'Abidjan et son état de pollution actuelle.

\section{MATARIEL ET METHODES}

Présentation de la zone d'étude

La zone d'étude est comprise entre $4^{\circ} 10$ et $5^{\circ} 30$ de latitude Nord et $3^{\circ} 50$ et $4^{\circ} 10$ de longitude Ouest (Figure 1). La ville 
d'Abidjan, capitale économique de la Côte d'Ivoire, est située sur la façade maritime en bordure de la lagune Ebrié. Elle s'étend sur une superficie de 58000 ha dont 9000 ha de lagunes soit $16 \%$ et 49000 ha de terre ferme soit 84\%. Depuis 1978, elle comprend dix communes (Abobo, Adjamé, Attécoubé, Cocody, Koumassi, Marcory, Plateau, PortBouët, Treichville, Yopougon) différentes les unes des autres par le mode d'occupation du sol, le niveau de vie des populations et les activités commerciales et industrielles (BNETD, 1996). L'urbanisation rapide de la ville a favorisé la création de plusieurs types d'habitats dont les habitats cours communes, individuels, collectifs et précaires ou spontanés. Ce dernier type ne bénéficie pas de systèmes adéquats et appropriés d'assainissement et d'approvisionnement en eau potable.

\section{Climat}

La ville d'Abidjan est couverte par un climat de transition dont le cycle annuel se divise en quatre saisons marquées par deux saisons pluvieuses et deux saisons sèches. Cette variation saisonnière climatique influence fortement la décomposition des ordures et intervient dans le processus de fermentation des déchets dont le lixiviat pourrait être entraîné en profondeur par les eaux d'infiltration. La hauteur moyenne annuelle des précipitations, déterminée sur la période 1963 à 2000, est de $1700 \mathrm{~mm}$ (Kouamé, 2007). Les eaux de pluie imbibent les déchets, composés à 50,6\% de matières organiques (Sané, 1999 in Sané, 2002), et les rendent plus lourds.

\section{Géologie et hydrogéologie}

Du point de vue géologique, la zone d'étude appartient au bassin sédimentaire côtier (d'âge crétacé-quaternaire) formé d'une alternance de couches de sables, d'argiles sableuses, d'argiles et de calcaires. D'un point de vue lithologique, les formations subissent latéralement et verticalement d'importantes variations de faciès.
Du point de vue hydrogéologique trois types de réservoirs aquifères, d'épaisseurs variables, peuvent être différenciés. Il s'agit de l'aquifère du Quaternaire, constitué de sables marins grossiers et de sables fins à grossiers. Son épaisseur est voisine de $50 \mathrm{~m}$. Cet horizon repose sur celui du Continental Terminal (CT) par l'intermédiaire de cuirasses latéritiques. Ce dernier d'âge Mio-Pliocène est fait de cuirasses latéritiques discontinues, de sables grossiers fluviatiles, d'argiles noires et sables argileux et de niveaux graveleux à passées d'argiles bariolées. Sa puissance (0 à $160 \mathrm{~m}$ au maximum) dépend de la profondeur du substratum et de l'état d'érosion de la surface du sol. Le troisième aquifère est celui du Crétacé supérieur (Maestrichtien) situé à environ $200 \mathrm{~m}$ de profondeur. Les formations du Maestrichtien possèdent des bancs calcaires peu fissurés, gréseux à gros grains de quartz roulés surmontant des sables fins coquilliers. Un forage exécuté par la Société des Eaux Minérales (SADEM), d'une profondeur de $190 \mathrm{~m}$, atteint cette formation. Par ailleurs, des études géophysiques réalisées par Faillat et al. (1979) ont donné une épaisseur de $40 \mathrm{~m}$ à Locodjro. L'épaisseur du Maestrichtien augmente vers le Sud.

L'existence des nappes contenues dans ces aquifères a été mise en évidence par les nombreuses sources qui les drainent, les cours d'eau et les forages qui les captent (Aghui et Biémi, 1984). Toutefois, seule la nappe du Continental Terminal qui fait l'objet de cette étude est exploitée pour l'alimentation en eau potable de la population d'Abidjan du fait de son extension régionale.

$\mathrm{Au}$ plan structural, ce bassin est traversé par une faille dite "faille des lagunes" d'orientation Est-Ouest présentant un pendage sud et un rejet pouvant atteindre 3500 mètres. Cette faille divise le bassin en deux parties distinctes (Aghui et Biémi, 1984) : au Nord, les sédiments de recouvrement d'épaisseur moyenne $300 \mathrm{~m}$ et au Sud, un fossé d'effondrement profond où le socle s'enfonce à 4000 ou $5000 \mathrm{~m}$. Le substratum présente une morphologie particulièrement accidentée avec des surcreusements qui sont 
des dépressions débouchant sur la lagune par des baies, des pointements de socle observables entre les paléovallées $(-40 \mathrm{~m}$ à l'Ouest d'Adiapo, -42,8 m à Yopougon) (Faillat et al., 1979 ; Aghui et Biémi, 1984).

\section{Actualisation des données socio- démographiques}

Les données socio-démographiques ont été actualisées à partir des données de base du recensement de la population de 1988 et 1998. Elles ont permis de calculer les taux de croissance moyen par commune utilisés dans l'estimation du nombre d'habitants par type d'habitats et par commune. Le regroupement du nombre d'habitants par type d'habitat et par niveau de vie a été réalisé suivant la nouvelle typologie de l'habitat proposé par le BNETD (Tableau 1). Les équations utilisées (Dongo, 2001), ci-après, ont permis de faire une estimation de la population à différents horizons.

$\mathrm{P}_{\mathrm{n}}=\mathrm{P}_{\mathrm{m}}(1+\mathrm{r})^{\mathrm{n}-\mathrm{m}} \quad \mathrm{r}=\left(\left(\mathrm{P}_{\mathrm{n}} / \mathrm{P}_{\mathrm{m}}\right)-1\right)^{1 / \mathrm{n}-\mathrm{m}}$

Avec $\mathrm{P}_{\mathrm{n}}$ : Population de l'année $\mathrm{n}$ (à estimer); $\mathrm{P}_{\mathrm{m}}$ : Population de l'année $\mathrm{m}$ (connue); $\mathrm{r}$ : taux de croissance moyen par commune.

Les données socio-démographiques actualisées nous ont permis de calculer les quantités de déchets domestiques genérées par les ménages.

\section{Quantification des déchets domestiques}

La quantification des ordures ménagères a été faite à l'aide des formules cidessous après actualisation des taux spécifiques de la production journalière par habitant. Cette actualisation est faite selon l'hypothèse émise dans le plan directeur de récupération et d'élimination des déchets solides de la ville d'Abidjan par le Cabinet Roche (1987) qui estime que la production spécifique des ordures ménagères devrait augmenter en moyenne de $1 \%$ par an et par niveau de vie. Ces ratios sont actualisés et appliqués au nombre d'habitants pour chaque niveau de vie.

La production globale $\mathrm{P}_{\mathrm{i}}$ des ordures en tonnes par an est donnée par la formule (2) suivante : $\mathrm{P}_{\mathrm{i}}=\left(365_{\mathrm{x}} \mathrm{P}_{\mathrm{x}} \mathrm{T}\right) / 1000$
Avec $\mathrm{P}_{\mathrm{i}}$ : Production globale des ordures en tonnes de l'année i ; P : Population estimée ; $\mathrm{T}$ : Taux de production spécifique en $\mathrm{Kg} / \mathrm{hab}$./jour.

Les quantités d'ordures non ramassées ont été estimées à partir des quantités d'ordures collectées et mises en décharge selon les données allant de 1995 à 2001 du BNETD.

$\mathrm{Q}=\left(\mathrm{P}_{\mathrm{i}}-\mathrm{Q}_{\mathrm{e}}\right)$

avec $\mathrm{Q}$ : Quantité d'ordures non ramassées ;

$\mathrm{Q}_{\mathrm{e}}$ : Quantité d'ordures enlevées ou collectées

La quantification des volumes d'eaux usées et de la charge polluante produits par les ménages a été calculée à partir des quantités d'eau potable consommées par les populations. Les ratios de consommation d'eau potable et rejets spécifiques correspondants sont indiqués également dans le Tableau 1. Ces paramètres sont fonction du niveau de vie des populations. Les rejets spécifiques constituent le facteur multiplicateur qui, appliqué aux consommations spécifiques en eau potable et au nombre d'habitants, permet de déterminer les quantités d'eaux usées produites par les ménages. La charge polluante des eaux usées domestiques est évaluée à partir des paramètres telles que la demande biologique en oxygène $\left(\mathrm{DBO}_{5}\right)$ et la demande chimique d'oxygène (DCO). L'étude menée par NEDECO (1981), dans les quartiers d'Abidjan, a revélé que par jour, les quartiers résidentiels généraient $35 \mathrm{~g}$ de $\mathrm{DBO}_{5}$, contre $30 \mathrm{~g}$ dans les quartiers économiques et $25 \mathrm{~g}$ dans les quartiers évolutifs et spontanés. Ces ratios restent encore valables et ont été utilisés dans cette étude.

Les volumes et la charge polluante des eaux usées rejetées dans la nature, hors des systèmes d'évacuation, ont été calculés selon le mode adopté par BCEOM (1984). En effet, BCEOM dans son étude estime que dans la conception générale des systèmes d'assainissement dans le contexte africain les volumes et charges polluantes rejetés à l'égout sont estimés à $40 \%$ de la production totale, soit $60 \%$ de la production des eaux usées domestiques. Ce taux n'ayant subi aucune modification à ce jour, a servi à calculer les 
quantités d'eaux usées domestiques et la charge polluante rejetée hors et dans le système d'égout pour l'ensemble de la ville d'Abidjan.

\section{Evaluation de la composition chimique de la nappe du Continental Terminal}

L'évaluation de l'état actuel de la composition chimique de la nappe a porté sur les résultats d'analyses chimiques réalisées au pas de temps mensuel, pendant la période allant de 1995 à 2001, par la Société de Distribution des Eaux de Côte d'Ivoire (SODECI). Il s'agit des eaux provenant des champs de captage de la ville d'Abidjan (Anonkoua Kouté, Abobo Avocatier, Zone Nord, Adjamé, Zone Ouest, Zone Est, Rivière Centre, Plateau, Nord Riviéra et de Niangon Nord) soit au total 57 forages retenus qui captent la nappe du CT. Ces résultats ont permis de faire une analyse spatio-temporelle des paramètres physico-chimiques des eaux du CT. En plus une étude statistique, portant sur l'analyse en composantes principales (ACP) et la classification ascendante hiérarchique $(\mathrm{CAH})$, a été réalisée sur les données chimiques de 1999 qui sont les plus complètes. La méthode de l'ACP a été utilisée dans l'optique d'identifier les liens qui existeraient entre les éléments chimiques (Travi et Mudry, 1997) et de déterminer les paramètres de pollution qui contribueraient à leur différenciation. L'application de cette méthode a mis à contribution les paramètres chimiques analysés (variables) et les observations (forages échantillonnés). La classification ascendante hiérarchique (CAH) qui est une méthode de regroupement a été réalisée à partir du même tableau de données utilisé dans l'ACP. La CAH permet d'obtenir un dendrogramme qui classe les observations ou les variables par groupes ou sous-groupes ayant le même poids ou les mêmes caractéristiques. Le critère d'agrégation est celui du moment centré d'ordre 2 qui minimise à chaque pas de calcul la variance intra classe de la partition construite (Guiral et Ferhi, 1989).
Enfin, l'apport de la cartographie et des techniques spatiales, intégrant l'occupation du sol, ont été essentielles dans l'élaboration des cartes de distribution spatiale des nitrates et des phosphates qui demeurent les paramètres chimiques dont les teneurs dans certains ouvrages ont atteint des cotes d'alerte.

\section{RESULTATS \\ Evolution démographique de la ville d'Abidjan}

Les données socio-démographiques indiquent que la population abidjanaise est passée de 2878200 habitants en 1998 à 3 883335 habitants en 2005, soit un taux de croissance de 4,37\%. Elle est inégalement répartie entre les différentes communes. Deux groupes de communes se dégagent suivant l'évolution du nombre d'habitants. Le premier groupe, représente les communes où l'augmentation de la population est significative (Yopougon, Abobo, Cocody et Koumassi ) et le deuxième groupe représente les communes dont l'évolution de la population est moins marquée (Treichville, Adjamé, Attécoubé, Marcory et Port bouët). Par ailleurs, Yopougon (688 522 habitants en 1998 et 1054577 habitants en 2005) et Abobo (638 203 habitants en 1998 et 883156 habitants en 2005) demeurent les plus peuplées avec à elles seules près de $50 \%$ de la population abidjanaise. Quant à la population du Plateau (10 368 habitants en 1998 et 9360 habitants en 2005), elle regresse et cela s'explique par le développement des centres commerciaux, des bureaux administratifs publics et privés au détriment des habitats (individuels ou collectifs).

\section{Quantification des ordures domestiques et des eaux usées}

La production moyenne d'ordures ménagères par habitant était de $0,84 \mathrm{~kg} / \mathrm{j}$ en 1998 et par la suite a atteint $0,91 \mathrm{~kg} / \mathrm{j}$ en 2005 . Cette production augmente avec le niveau de vie des populations. La production globale des ordures ménagères est passée de 994043 tonnes en 1998 à 1438779 tonnes en 2005 (Tableau 2). Les communes de grande 
production d'ordures sont celles de Yopougon, Abobo, Cocody, Koumassi et Adjamé avec une production annuelle individuelle supérieure à 90000 tonnes pour chaque commune depuis 1998. La composition des ordures ménagères, en fonction du niveau de vie des populations, de la ville d'Abidjan est indiquée dans le Tableau 3.

Parallèlement à cette production d'importantes quantités de déchets ménagers, estimées à 475172 tonnes ne sont pas ramassées pour l'ensemble des communes en 1998. En 2005, ces déchets non ramassés ont atteint 548684 tonnes sans le cumul des quantités non ramassées des années précédentes.

Les quantités journalières d'eaux usées rejetées par la population dans chaque commune sont reportées dans le Tableau 4. La production de la ville d'Abidjan est passée de $162323 \mathrm{~m}^{3}$ en 1998 à $193186 \mathrm{~m}^{3}$ en 2002. Cette production varie d'une commune à une autre. Les communes de grandes productions sont Yopougon, Abobo, Cocody, Koumassi, Adjamé, Port-Bouët, Attecoubé et Marcory, dont la production individuelle est estimée à plus de $10000 \mathrm{~m}^{3}$ par jour depuis 1998, soit $3650000 \mathrm{~m}^{3}$ par an.

\section{Charge polluante des eaux usées domestiques}

Les charges polluantes des eaux usées domestiques de la ville d'Abidjan sont consignées dans le Tableau 4. La charge journalière moyenne est estimée à $91198 \mathrm{Kg}$ de $\mathrm{DBO}_{5}$, soit 33287 tonnes de $\mathrm{DBO}_{5}$ par an. Les eaux les plus chargées proviennent des communes de Yopougon, Abobo, Cocody et Koumassi et Adjamé avec en moyenne une production journalière supérieure à $7000 \mathrm{~kg}$ de $\mathrm{DBO}_{5}$. Les rejets dans l'égout et hors de l'égout, estimés et indiqués dans le Tableau 4 montrent qu'entre 2002 et 2005 , les rejets hors de l'égout sont passés de $115913 \mathrm{~m}^{3}$ à $132533 \mathrm{~m}^{3}$ par jour avec des charges correspondantes estimées respectivement à $56113 \mathrm{Kg}$ et $64047 \mathrm{Kg}$ de $\mathrm{DBO}_{5}$ par jour.
Qualité des eaux souterraines et risques de pollution de la nappe du Continental Terminal

Les résultats obtenus à partir de l'ACP permettent d'identifier les facteurs de pollution qui influencent le chimisme des eaux de la nappe du CT et qui sont à l'origine de leur différenciation. La représentation des données dans le plan factoriel F1-F2 exprime $62,97 \%$ de la variance totale du nuage de points dont $46,83 \%$ pour le facteur F1(Figure 2). Les paramètres physico-chimiques: $\mathrm{CE}$, TDS, THT, THCa, NH4, $\mathrm{Cl}$ et $\mathrm{K}$ sont bien corrrélés entre eux et présentent un poids significatif dans la prévalence du phénomène représenté par F1. Les éléments CE, TDS sont caractéristiques de la minéralisation naturelle de l'eau dans son encaissant (Mudry et Blavoux, 1986), tandis que $\mathrm{Cl}$ et $\mathrm{NH} 4$ traduisent une minéralisation induite. Dans ce cas cet axe exprime le temps de séjour des éléments; c'est un indicateur de la minéralisation (Hani et al., 1997). L'axe F2, avec $16,14 \%$ de l'inertie totale, est déterminé par $\mathrm{SiO}_{2}$ mais également par $\mathrm{NO}_{3}, \mathrm{Al}$ et $\mathrm{NO}_{2}$. Ces paramètres sont inversement corrélés à $\mathrm{pH}, \mathrm{SO}_{4}$ et à un degré moindre à $\mathrm{T}^{\circ}$. Ce facteur rend compte des conditions du milieu et de leur influence sur les variations chimiques des eaux ; F2 représente les apports superficiels. C'est également un indicateur d'une minéralisation naturelle $\left(\mathrm{SiO}_{2}\right)$ et induite $\left(\mathrm{NO}_{3}\right)$.

Ces regroupements ne s'individualisent pas nettement sur le dendrogramme de la Figure 3 dans lequel on peut cependant identifier trois groupes de points. Le premier groupe est celui formé par les paramètres CE et TDS qui déterminent le dégré de minéralisation des eaux. Le second comprend en partie les composés azotés dont les proportions relatives $\left(\mathrm{NO}_{3}\right.$ et $\left.\mathrm{NO}_{2}\right)$ sont fonction de la température. Enfin, le troisième groupe est représenté par les sels minéraux $\left(\mathrm{Cl}, \mathrm{SiO}_{4}, \mathrm{SiO}_{2}, \mathrm{~K}, \mathrm{Al}, \mathrm{THCa}\right.$, THMg, THT) dont l'apparition est à mettre en relation avec la diversité des processus de minéralisation fortement dépendant $\mathrm{du} \mathrm{pH}$ des eaux. Le Tableau 5 qui résume les teneurs 
moyennes de quelques paramètres physicochimiques de la nappe du CT, mesurées au cours de la période allant de 1995 à 2001, permet d'apprécier l'état de la qualité des eaux souterraines dans les différentes zones de la ville d'Abidjan. Les valeurs de conductivité sont comprises entre 7 et $315 \mu \mathrm{Scm}^{-1}$ traduisant une faible minéralisation des eaux de la nappe. Ces eaux présentent une forte pollution nitratée avec des valeurs atteignant souvent $120 \mathrm{mg} \mathrm{L}^{-1}$. Les concentrations excessives en composés azoté, phosphaté et métallique (supérieures aux normes OMS) sont relevées particulièrement dans les secteurs du Plateau, d'Adjamé et de la Zone Ouest (Figures 4 et 5). Les secteurs d'Anonkoua Kouté, Niangon Nord et Nord Riviera sont également affectés par une pollution métallique et phosphatée. Des variations importantes y sont observées dans l'évolution temporelle des concentrations en nitrates, phosphates et aluminium dans les secteurs de captage. Ainsi l'évolution du pourcentage de forages contaminés par les nitrates entre 1999 et 2001 est de $18 \%$ en 1999, $9 \%$ en 2000 et $14 \%$ en 2001 . Pour l'ensemble des communes, $59,6 \%$ des forages présentaient en 1999 des teneurs en phosphates supérieures à $0,05 \mathrm{mg} \mathrm{L}^{-1}$. Ce pourcentage est passé à $87,7 \%$ en 2000 ensuite $89,5 \%$ en 2001. Enfin en 1999, sur un effectif de 57 forages, $14 \%$ présentaient des teneurs supérieures aux normes OMS (0,2 mg $\mathrm{L}^{-1}$ ) requises pour l'aluminium puis ce nombre a chuté à $10 \%$ en 2000 et 2001. De tels pourcentages sont très alarmants.

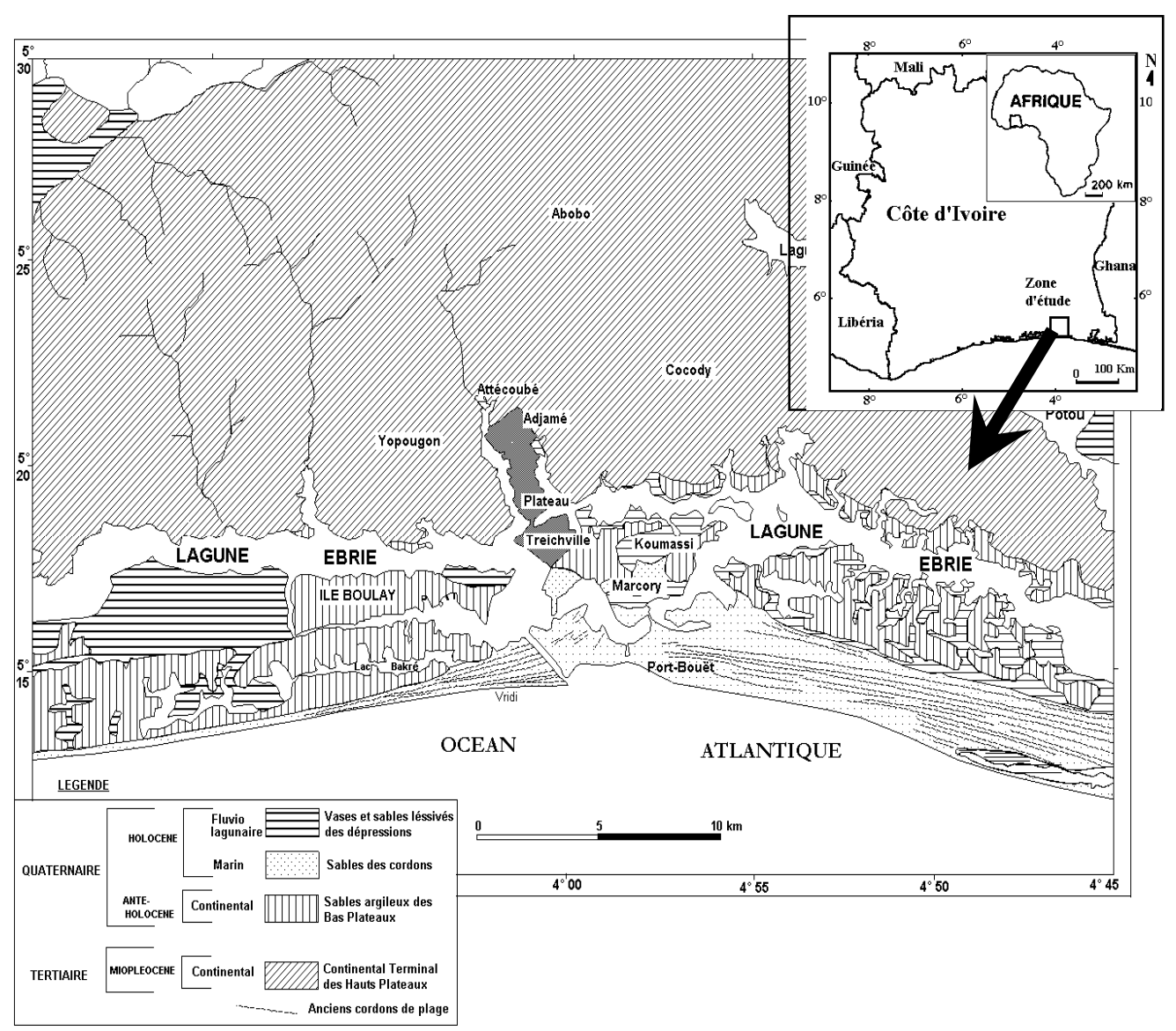

Figure 1 : Carte de localisation de la zone d'étude 


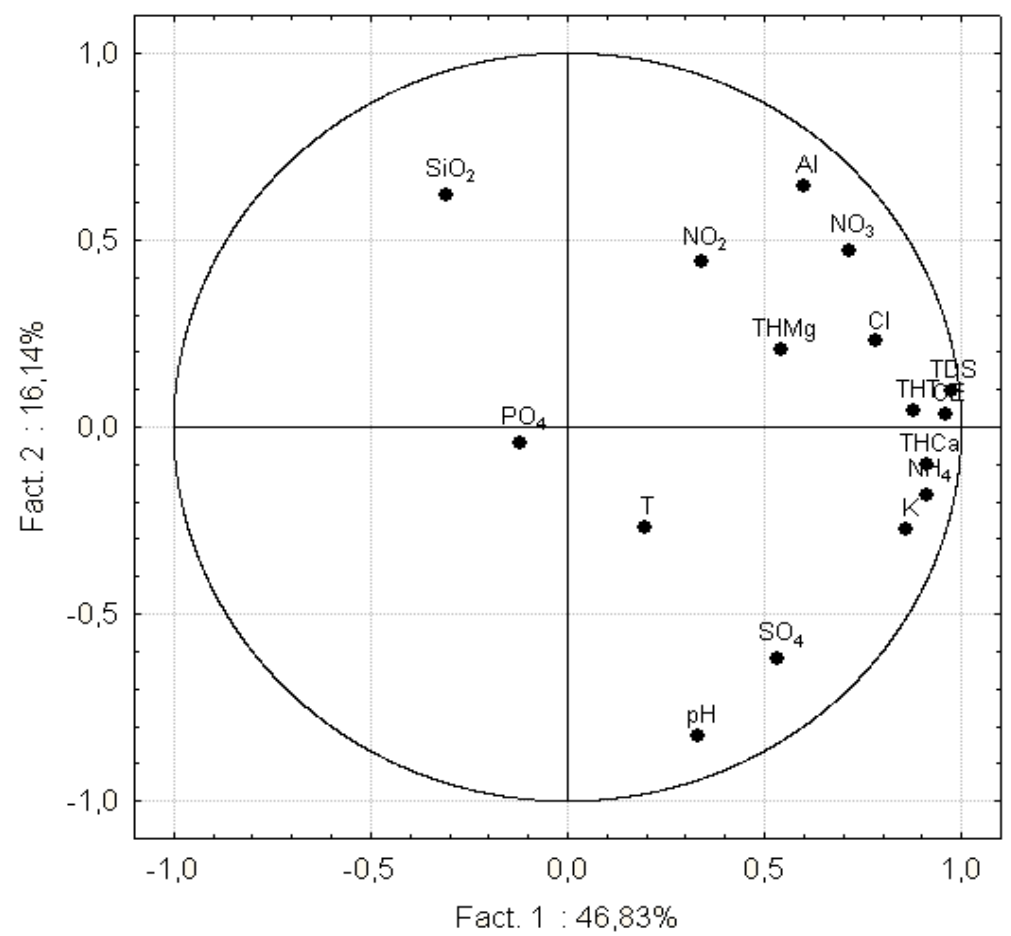

Figure 2 : Analyse en Composantes principales des données chimiques des eaux de la nappe d'Abidjan. Projection dans le plan factoriel F1 x F2.

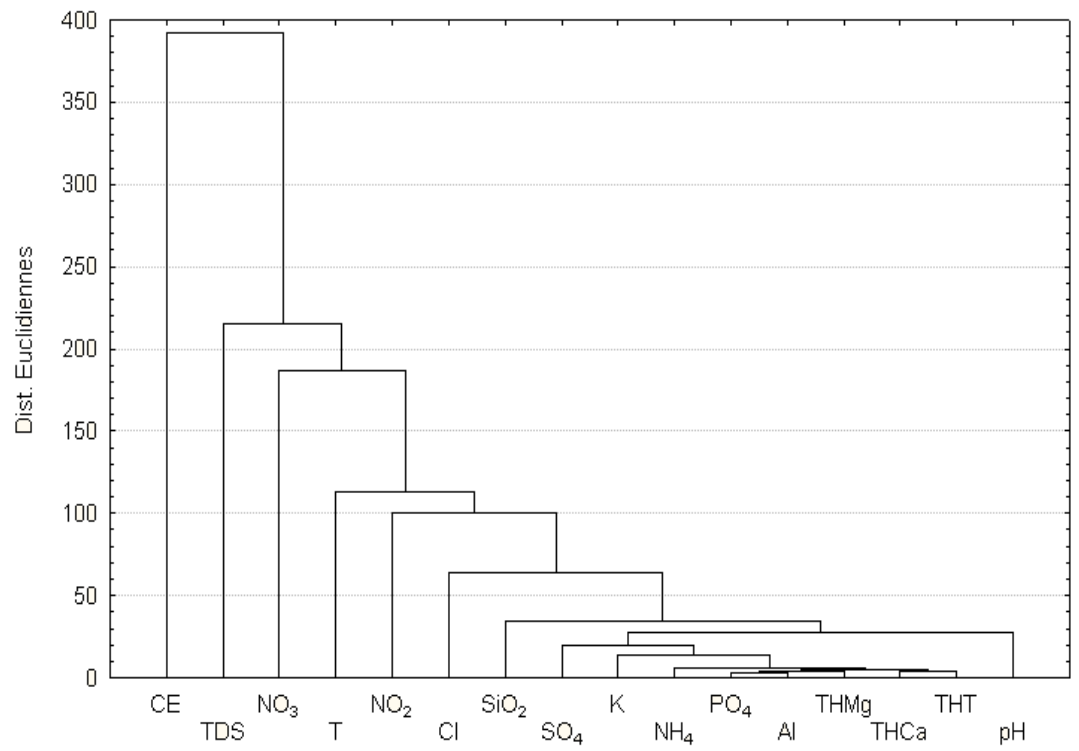

Figure 3 : Dendrogramme de classification hiérarchique des données chimiques des eaux de la nappe d'Abidjan. 

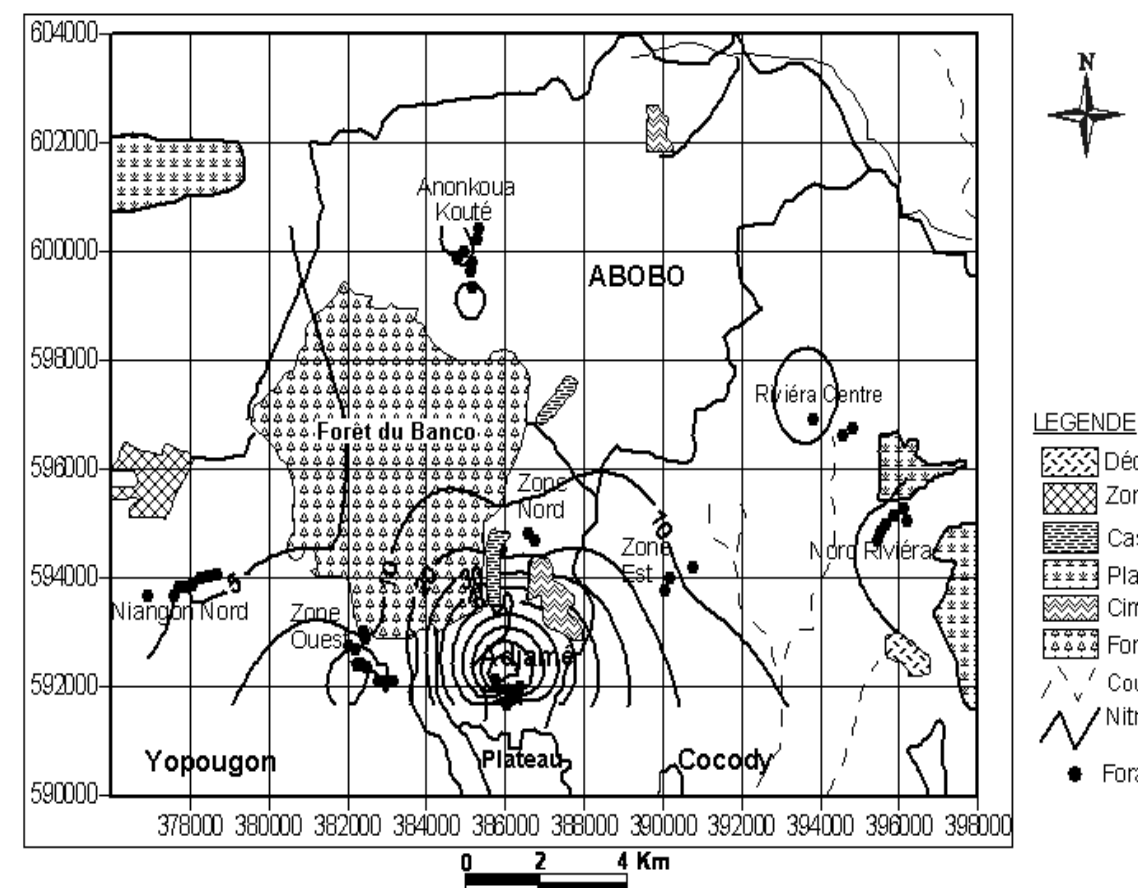

LEGENDE

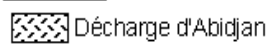

Zone industrielle

度至Casses d'Abidjan

Plantations

Cimétières

404 Forêt

$>$ 'Cours d'eau

$\bigwedge^{N}$ Nitrates en mg.L-1

- Forages

a- Année 2000

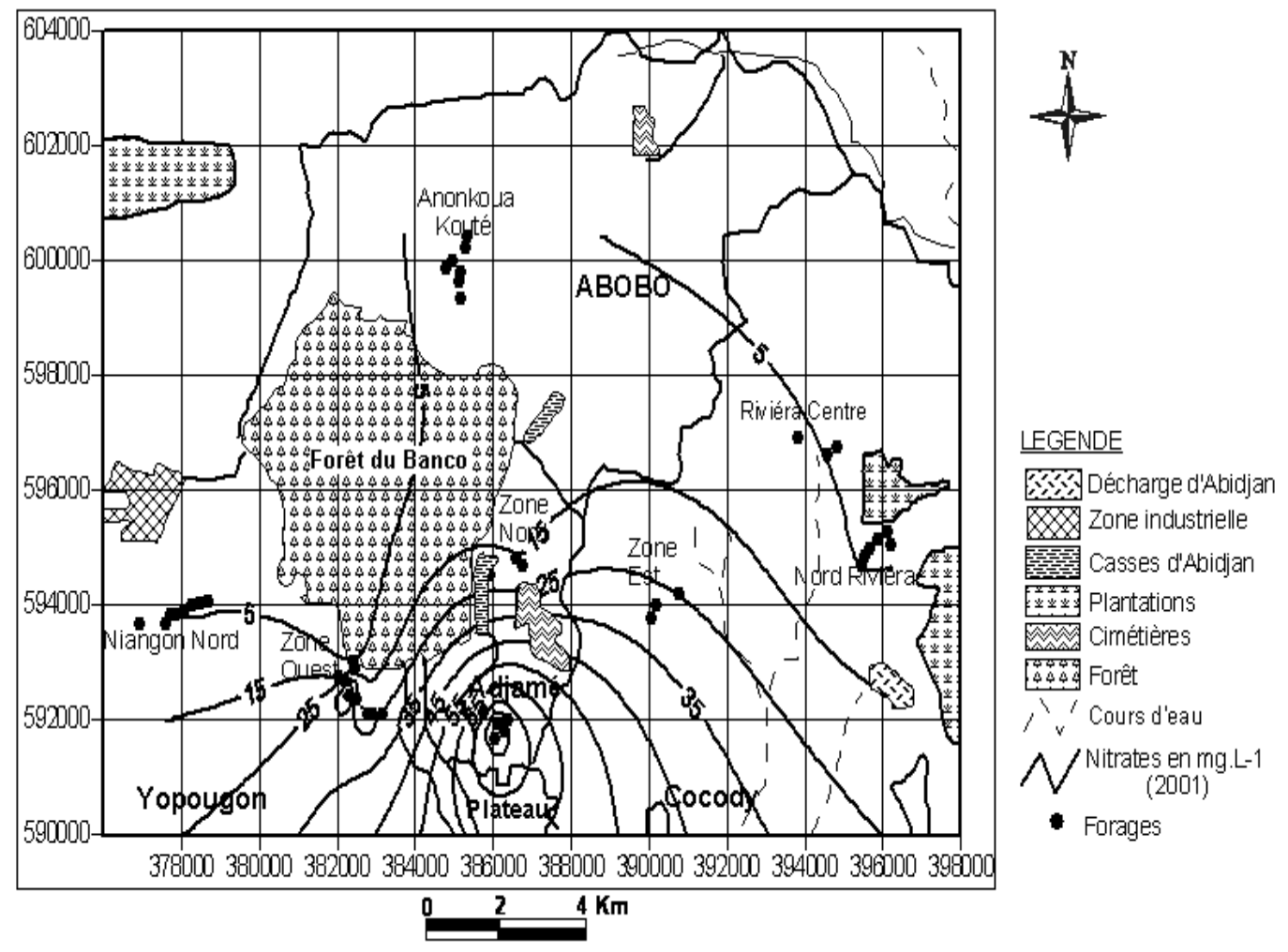

b- Année 2001

Figure 4 : Carte de distribution spatiale des teneurs en nitrates dans la nappe d'Abidjan. 

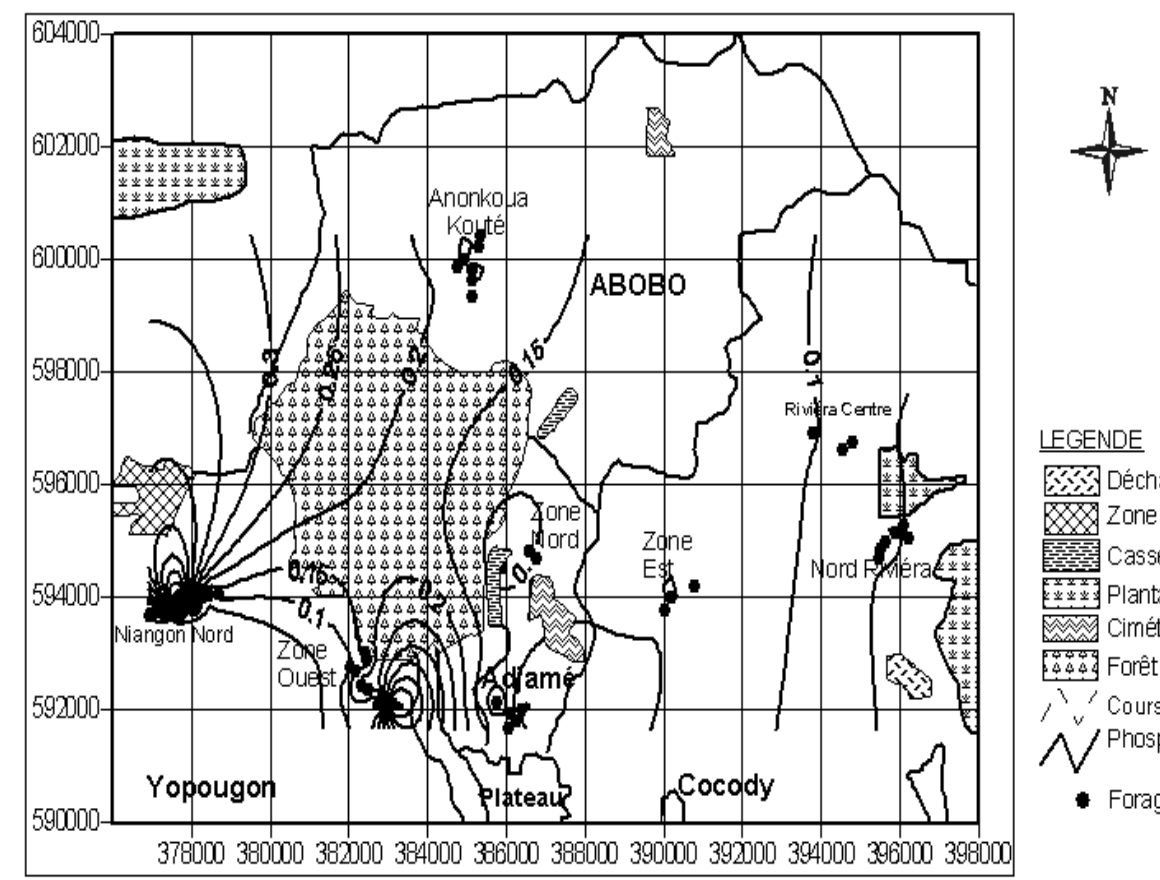

LEGENDE

窗沟Décharge d'Abidjan

Zone industrielle

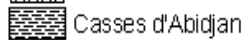

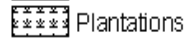

Cimétières

44404 Forêt

'Cours d'eau

$\wedge^{1}$ Phosphates en mg. $\mathrm{L}^{-1}$

$$
\text { - Forages }
$$

\section{a- Année 2000}
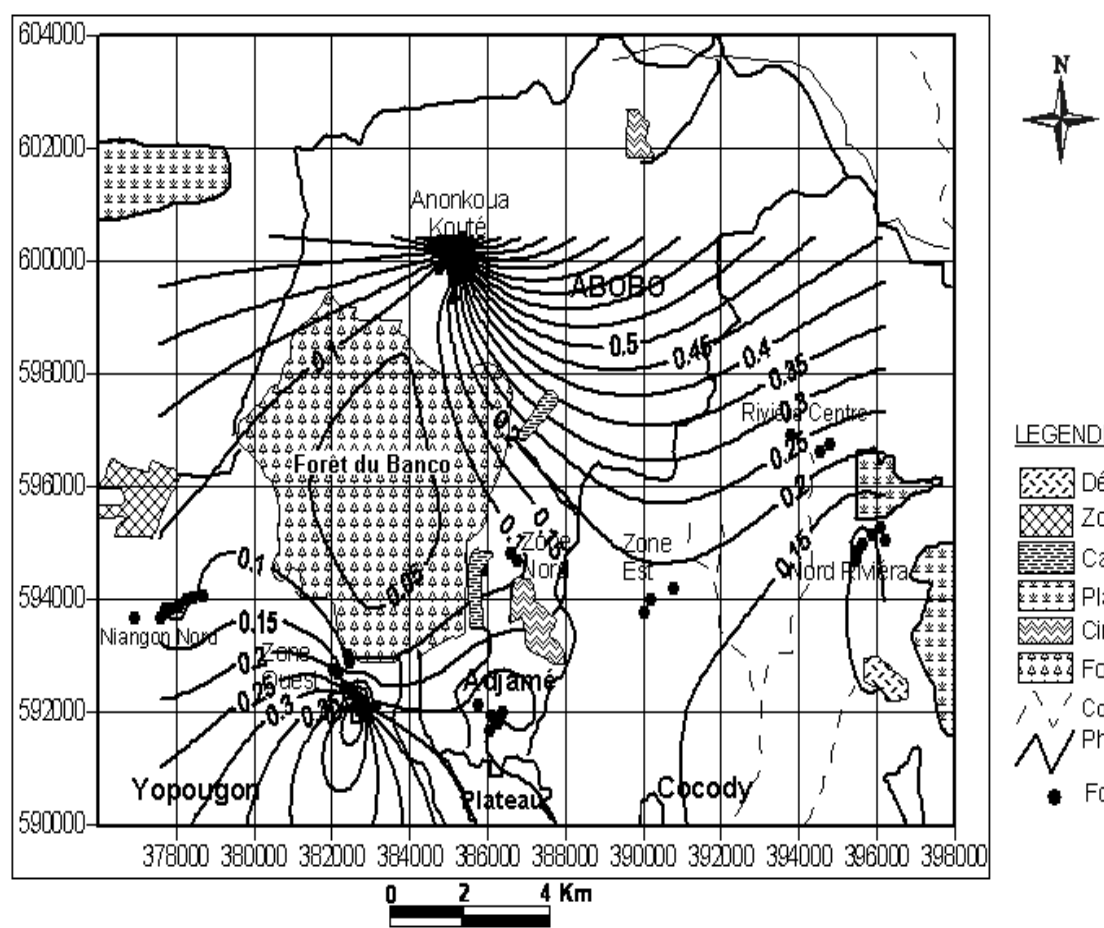

LEGENDE

空家Décharge d'Abidjan

Zone industrielle

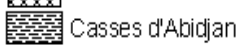

Plantations

Cimétières

404 Forêt

1 'Cours d'eau

$\wedge^{v}$ Phosphates en mg. $\mathrm{L}^{-1}$

- Forages

b- Année 2001

Figure 5 : Carte de distribution spatiale des teneurs en phosphates dans la nappe d'Abidjan. 
Tableau 1 : Typologie de l'habitat, niveau de vie, consommation et pollution spécifiques.

\begin{tabular}{|c|c|c|c|c|}
\hline $\begin{array}{l}\text { Ancienne } \\
\text { typologie }\end{array}$ & Nouvelle typologie & Niveau de vie & $\begin{array}{l}\text { Consommation } \\
\text { spécifique en } \\
\text { eau potable } \\
(\mathrm{L} / \mathrm{hab} / \mathbf{j})\end{array}$ & $\begin{array}{c}\text { Taux de rejet } \\
\text { spécifiques } \\
\text { des eaux } \\
\text { usées } \\
\text { (L/hab/jour) }\end{array}$ \\
\hline $\begin{array}{l}\text { Habitat } \\
\text { résidentiel }\end{array}$ & $\begin{array}{l}\text { Habitat individuel bon } \\
\text { standing }\end{array}$ & Haut niveau de vie & 340 & 0,45 \\
\hline $\begin{array}{l}\text { Habitat } \\
\text { économique }\end{array}$ & $\begin{array}{l}\text { Habitat collectif moyen } \\
\text { standing } \\
\text { Habitat collectif bon standing } \\
\text { Habitat individuel économique } \\
\text { Habitat individuel moyen } \\
\text { standing } \\
\text { Habitat cour commune } \\
\text { Habitat collectif économique }\end{array}$ & $\begin{array}{l}\text { Niveau de vie } \\
\text { moyen }\end{array}$ & 90 & 0,80 \\
\hline Habitat spontané & Habitat précaire & $\begin{array}{c}\text { Faible niveau de } \\
\text { vie }\end{array}$ & 60 & \\
\hline
\end{tabular}

Tableau 2 : Quantités en tonnes d'ordures ménagères produites par commune.

\begin{tabular}{lccccc}
\hline Communes & $\mathbf{1 9 9 8}$ & $\mathbf{2 0 0 0}$ & $\mathbf{2 0 0 1}$ & $\mathbf{2 0 0 2}$ & $\mathbf{2 0 0 5}$ \\
\hline ABOBO & 230439,24 & 257962,89 & 272893,53 & 288660,53 & 341450,03 \\
ADJAMÉ & 91155,95 & 97625,16 & 101014,79 & 104511,88 & 115675,64 \\
ATTÉCOUBÉ & 71770,23 & 76846,07 & 79504,21 & 82245,69 & 90995,11 \\
COCODY & 92851,77 & 108228,01 & 116829,54 & 126103,02 & 158493,25 \\
KOUMASSI & 100603,06 & 110464,51 & 115729,83 & 121228,11 & 139254,35 \\
MARCORY & 64698,44 & 68661,81 & 70723,31 & 72839,81 & 79533,21 \\
PLATEAU & 3784,34 & 3748,91 & 3730,76 & 3712,37 & 3655,71 \\
PORT-BOUËT & 56216,65 & 60485,60 & 62723,13 & 65032,10 & 72409,04 \\
TREICHVILLE & 43449,50 & 45139,59 & 46002,32 & 46877,00 & 49574,23 \\
YOPOUGON & 239074,12 & 275722,03 & 296054,31 & 314478,46 & 387738,17 \\
Total & $\mathbf{9 9 4} \mathbf{0 4 3 , 3 0}$ & $\mathbf{1 1 0 4} \mathbf{8 8 4 , 5 8}$ & $\mathbf{1 1 6 5 2 0 5 , 7 3}$ & $\mathbf{1 2 2 5} \mathbf{6 8 8 , 9 7}$ & $\mathbf{1 4 3 8} \mathbf{4 7 8 , 7 4}$ \\
\hline
\end{tabular}

Tableau 3 : Composition (\%) des déchets ménagers de la ville d’Abidjan (Sané, 1999).

\begin{tabular}{lccccc}
\hline Matières & $\begin{array}{c}\text { Haut niveau } \\
\text { de vie }\end{array}$ & $\begin{array}{c}\text { Niveau de vie } \\
\text { moyen }\end{array}$ & $\begin{array}{c}\text { Niveau de vie } \\
\text { faible }\end{array}$ & $\begin{array}{c}\text { Commerces } \\
\text { et affaires }\end{array}$ & Industries \\
\hline Fermentescibles & 52,69 & 50,55 & 49,69 & 25,32 & 10,17 \\
Végétaux & 15,34 & 19,18 & 13,89 & 7,47 & 5,47 \\
Fines & 8,04 & 12,95 & 25,19 & 11,15 & 33,74 \\
Plastiques & 8,29 & 7,25 & 4,70 & 10,82 & 14,66 \\
Papiers & 7,11 & 5,82 & 4,32 & 36,66 & 16,31 \\
Textiles & 5,26 & 2,17 & 0,94 & 4,12 & 8,40 \\
Métaux & 1,49 & 0,95 & 0,55 & 1,61 & 7,93 \\
Cailloux & 0,71 & 0,66 & 0,38 & 0 & 1,30 \\
Verre & 1,07 & 0,48 & 0,33 & 2,84 & 2,02 \\
Total & $\mathbf{1 0 0}$ & $\mathbf{1 0 0}$ & $\mathbf{1 0 0}$ & $\mathbf{1 0 0}$ & $\mathbf{1 0 0}$ \\
\hline
\end{tabular}


Tableau 4 : Rejets d'eaux usées et charge polluante hors de l'égout d'Abidjan $\left(\mathrm{Kg} \mathrm{DBO}_{5} / \mathrm{j}\right)$.

\begin{tabular}{lcccccc}
\hline \multirow{2}{*}{ Années } & \multicolumn{2}{c}{ Eaux usées domestiques en $\mathbf{~ m}^{3} / \mathbf{j}$} & \multicolumn{2}{c}{ Pollution en Kg DBO } & (j/j \\
\cline { 2 - 7 } & Production & Egout & Hors égout & Production & Egout & Hors égout \\
\hline 1998 & 162323 & 64929 & 97394 & 78757 & 31503 & 47254 \\
2000 & 176966 & 70786 & 106180 & 85767 & 34307 & 51460 \\
2002 & 193186 & 77274 & 115912 & 93521 & 37408 & 56113 \\
2005 & 220888 & 88355 & 132533 & 106745 & 42698 & 64047 \\
\hline
\end{tabular}

Tableau 5 : Teneurs moyennes de quelques paramètres physico-chimiques $(\mathrm{mg} / \mathrm{L})$ des eaux de forages de la ville d'Abidjan (1999-2001).

\begin{tabular}{lcccccccc}
\hline Stations & $\mathbf{N O}_{\mathbf{3}}$ & $\mathbf{N O}_{\mathbf{2}}$ & $\mathbf{N H}_{\mathbf{4}}$ & $\mathbf{P O}_{\mathbf{4}}$ & $\mathbf{S O}_{\mathbf{4}}$ & $\mathbf{C l}$ & $\mathbf{A l}$ & ${ }^{*}$ Cond \\
\hline Plateau & 120 & 0,03 & 3,47 & 0,17 & & 54,10 & 0,48 & 239 \\
Adjamé & 65 & 0,52 & 6,49 & 1,42 & 19,5 & 22,15 & 1,02 & 314 \\
Zone Nord & 8 & 0,03 & 0,03 & 0,14 & & 6,94 & 0,03 & 7,1 \\
Zone Ouest & 120 & 0,03 & 1,58 & 0,18 & 1,0 & 15,5 & 0,78 & 106,4 \\
Anonkoua Kouté & 10 & 0,03 & 0,05 & 0,14 & 0,3 & 4,48 & 0,18 & 48 \\
Nord Riviera & 3,0 & 0,02 & 0,02 & 0,85 & 0,4 & 7,03 & 0,00 & 38,6 \\
Zone Est & 0,3 & 0,04 & 0,02 & 0,37 & 16,0 & 5,0 & 0,06 & 82,5 \\
Riviera Centre & 5,0 & 0,03 & 0,07 & 0,10 & & 4,33 & 0,05 & 41,3 \\
Niangon Nord & 4,0 & 0,06 & 0,02 & 1,01 & 0,6 & 0,25 & 0,17 & 38,7 \\
Normes $\boldsymbol{O M S}$ & 50 & 0,1 & 0,05 & 0,5 & 250 & 200 & 0,2 & 400 \\
\hline *Conductivité exprimée en $\mu \mathrm{S} / \mathrm{cm}$ & & & & & & &
\end{tabular}

\section{DISCUSSION}

Impact de la gestion des déchets municipaux sur la qualité des eaux

Les résultats socio-démographiques obtenus montrent que la croissance de la population de l'agglomération d'Abidjan a entraîné une augmentation logique de la production des déchets urbains non ramassés et l'insuffisance de la gestion des municipalités. La typologie de l'habitat est très diversifiée. Elle montre que c'est surtout dans les quartiers à niveau de vie élevé qu'on a la plus forte production d'ordures ménagères par habitant de l'ordre de 1,66 kg/j. Certaines communes possèdent encore un système d'assainissement peu développé, et le rejet des déchets se fait directement dans les égouts, souvent à ciel ouvert qui débouchent dans la lagune Ebrié. Le rôle récepteur de la lagune Ebrié a été évoqué par de nombreux auteurs (Broche et Peschet, 1983; Koné et Aka, 1996) qui ont montré que toutes les industries de l'agglomération d'Abidjan y déversaient leurs eaux usées estimées à $12000 \mathrm{~m}^{3} / \mathrm{j}$. Par ailleurs, l'absence d'une politique de valorisation par le recyclage de ces déchets a un impact certain sur la qualité du cadre de vie. Les communes les plus engorgées par ces déchets non collectés sont Yopougon qui est envahie par $35 \%$ de l'ensemble des ordures 
ménagères non ramassées, Abobo (22\%), Cocody (14\%), Attécoubé (9\%), Koumassi $(7 \%)$ et Adjamé (6\%). Ainsi, dans ces communes, les quantités de déchets stockées en permanence constituent des dépôts sauvages qui prolifèrent dans les zones d'habitats, dans les ravins, les caniveaux et les puits abandonnés. Le lixiviat produit par ces déchets est ruisselé vers les eaux de surface (eaux lagunaires en particulier) ou s'infiltre vers la nappe phréatique située à moins de 20 $\mathrm{m}$ de profondeur dans certains quartiers (Kouadio et al., 2000 ; Adjiri et al., 2008). Ces déchets non ramassés sont à l'origine de la propagation des agents vecteurs de maladies tels que le paludisme, la fièvre typhoïde, le choléra, les maladies diarrhéiques, etc. Selon WHO (2005) in Dongo et al., (2008), l'épidémie de fièvre jaune qui s'est abattue sur Abidjan, en 2005, a fait des victimes dans les quartiers précaires de la ville, y compris ceux de Yopougon. En terme de rejet d'eaux usées, la commune de Yopougon fait partie de celles où le taux de rejet par habitant et par jour est élevé $(0,80 \mathrm{~L} / \mathrm{hab} / \mathrm{j})$. C'est également la plus peuplée d'Abidjan et celle qui produit la plus grande quantité d'ordures ménagères non ramassées (192 769,17 tonnes en 2002). Ces quantités importantes de déchets non ramassées qui jonchent les rues des quartiers sont sources d'importantes charges polluantes qui peuvent être évaluées en $\mathrm{DBO}_{5}$, en $\mathrm{DCO}$, en azote total, etc. Les ordures provenant des zones d'habitats résidentiels d'Abidjan produisent jusqu'à 5200 ppm de $\mathrm{DBO}_{5}$ contre 3300 ppm pour les zones d'habitat collectif (AGHTM, 1992). Les valeurs assez élevées de $\mathrm{DBO}_{5}$ indiquent une forte pollution organique issue de la dégradation de la matière organique. En effet, l'accumulation des ordures ménagères putrescibles non ramassées et des eaux usées rejetées hors égout, ces dernières années, soulignent bien l'ampleur des charges polluantes émises dans l'environnement. C'est au niveau des commerces qu'on enregistre les plus fortes valeurs de charges polluantes (7 $100 \mathrm{ppm}$ de
$\mathrm{DBO}_{5}$ ). Ceci se comprend aisément puisque ce secteur d'activité est surtout dominé par les industries agro-alimentaires et textiles et les eaux usées qui en sont issues sont composées de substances toxiques telles que la soude, les acides, les huiles minérales, les pigments des industries textiles et la glycérine des savonneries provenant des ateliers métallurgiques et de tannage artisanal. L'impact de ces rejets sur les eaux de surface est certain. Le lixiviat de la décharge d'Akouédo présente un niveau de pollution très élevé. La composante organique de cette pollution chimique est caractérisée par un rapport $\mathrm{DCO} / \mathrm{DBO}_{5}$ égal à 1,65 indiquant la prédominance d'une source domestique de cette pollution (Kouadio et al., 2000). Par ailleurs, les volumes d'eaux usées domestiques dont les charges polluantes sont importantes constituent des dangers pour les populations et les ressources en eaux. Ces eaux putrides qui sont chariées par les eaux de ruissellement, en direction des lagunes et des différents cours d'eau de la ville (Banco, Gbangbo, etc.), constituent une ménace pour les eaux souterraines en présence et partant de la chaîne alimentaire. A ce propos, signalons que ces eaux usées sont réutilisées dans l'agriculture périurbaine et particulièrement dans l'arrosage des cultures de maraîchage. Or il est établi que les nuisances causées par les effluents liquides sont plus dangereux, à la fois pour la santé humaine et pour l'environnement. A ce titre Koné et Aka (1996) ont signalé des cas de mortalités massives survenues en 1979 chez des crustacés, des poissons et des mollusques dans la baie de Toupah (système lagunaire Ebrié).

\section{Evolution spatio-temporelle de la pollution en composés azoté, phosphaté et métallique} Les différentes analyses projetées dans les plans factoriels F1-F2 montrent que la nappe d'Abidjan présente deux pôles d'aquisition de la minéralisation. Le premier pôle est une aquisition naturelle à partir des interactions eaux-roches encaissantes pendant 
le séjour des eaux dans l'aquifère. Le deuxième pôle est dû aux apports superficiels par infiltration des polluants urbains générés par les activités anthropiques. En effet, la contamination des eaux souterraines de certains ouvrages par les nitrates résulte de plusieurs sources: 1) l'oxydation et/ou décomposition de la matière organique liée aux activités humaines, agricoles ou urbaines. Les décharges incontrôlées constituent effectivement des sources potentielles de contamination des eaux souterraines en $\mathrm{NO}_{3}$. Dans le sol, la matière organique est minéralisée à partir de l'oxydation biologique de $\mathrm{NH}_{4}$ en $\mathrm{NO}_{3}$ (Tandia et al., 1999). Ainsi, les nombreuses cultures maraîchères pratiquées dans la ville d'Abidjan et à sa périphérie concourent à l'augmentation de la teneur en nitrates des eaux souterraines. 2) les eaux usées (domestique, industrielle et agricole) rejetées hors égout suite à la faible couverture du réseau d'assainissement et estimées à $60 \%$ de la production favoriseraient la pollution nitrique des eaux souterraines (Aghzar et al., 2002). 3) les dépôts sauvages d'ordures dans les quartiers constituent la troisième source de production de nitrates et par conséquent de pollution diffuse de la nappe par les nitrates issus du lessivage du sol (Tandia et al., 1997 ; Baali et al., 2007). Dans ce cas les variations spatiotemporelles des concentrations en nitrates observées, sont certainement le reflet des multiples sources de productions de $\mathrm{NO}_{3}$ à travers la ville d'Abidjan en liaison avec la densité de population et les quantités d'ordures qui en résultent. Les pics de pollution relevés en 1999 au Plateau, Adjamé et Zone Ouest, indiquent une contamination de la nappe par des nitrates d'origine organique. A Adjamé, la densité de la population y est très élevée en raison d'une proportion plus grande d'habitats cours communes et donc de la présence de nombreuses latrines. D'ailleurs, les travaux de Kouadio et al. (1998) signalent l'existence d'un ancien dépotoir aux voisinages du forage F8 de cette commune. Cette évolution de la pollution nitratée n'est plus circonscrite uniquement aux anciens quartiers. Elle s'étend maintenant vers les nouveaux pôles d'habitation des zones industrielles de Yopougon (Zone ouest). Par ailleurs, les fréquentes augmentations de $\mathrm{NO}_{3}, \mathrm{~K}$ et $\mathrm{PO}_{4}$ dans certaines eaux apparaissent liées à l'accroissement de l'urbanisation (Boukari et al., 1996). A Abidjan, la distribution spatiale des teneurs en $\mathrm{PO}_{4}$ dans la nappe est en évolution constante dans les secteurs de Niangon nord, d'Adjamé et de nord Riviera. L'élévation anormale de ces teneurs en $\mathrm{PO}_{4}$ (au dessus des normes OMS) dans ces deux derniers secteurs témoigne de la contamination de la nappe à partir soit d'une source de pollution (déchets domestiques, puits abandonnés, effluents industriels, percolation à partir de drains), soit d'une pollution diffuse (eaux de ruissellement). Ce type de pollution est un long processus dépendant largement du taux d'infiltration, des processus géochimiques tels que l'oxydoréduction et des réactions de surface (Boukari et al., 1996). Enfin, l'eutrophisation accélérée du plan d'eau lagunaire de la ville d'Abidjan résulte des fortes concentrations en éléments azotés et phosphatés contenus dans les lixiviats issus des différentes décharges et des baies (Adjiri et al., 2008). Les teneurs élevées en aluminium sont observées dans les secteurs d'Adjamé et de la Zone Ouest et plus ou moins au Plateau où les forages présentent des concentrations supérieures à $0,2 \mathrm{mg} \mathrm{L}^{-1}$. Dans le secteur d'Adjamé, seuls les forages F2 et F8 présentent des teneurs significatives en aluminium. Concernant le secteur de la Zone Ouest, seul le forage $\mathrm{ZO}_{6}$ a présenté des valeurs en aluminium supérieures à $0,5 \mathrm{mg} / \mathrm{L}$. Cet élément est faiblement présent dans les eaux de la nappe d'Abidjan. Son évolution est régressive puisque le nombre de forages dont les eaux présentent des teneurs supérieures à 0,2 mg/L est passé de 8 en 1999 à 6 en 2001. Cette diminution peut être attribuée à une dilution par les eaux de pluie, 
par contre les augmentations ponctuelles observées proviendraient des apports liés aux activités commerciales et industrielles.

\section{Conclusion}

La mauvaise gestion des déchets domestiques (eaux usées et ordures ménagères) est à l'origine du stockage permanent dans la ville d'Abidjan d'importantes quantités de déchets. Les systèmes d'évacuation des déchets de la ville sont défaillants. Les eaux usées domestiques déversées par jour hors des systèmes d'égout sont estimées à plus de $130000 \mathrm{~m}^{3}$ dont la charge polluante journalière induite est évaluée à $64000 \mathrm{Kg}$ de $\mathrm{DBO}_{5}$. Quant aux ordures ménagères, c'est plus de 500000 tonnes qui engorgent chaque année l'ensemble de la ville. Ces ordures ménagères se retrouvent dans les fosses, les dépressions naturelles, les canaux en terre, les puits abandonnés, etc. La minéralisation des eaux de la nappe se fait par deux voies. L'une fait intervenir les interactions eaux-roches encaissantes et l'autre est due aux apports superficiels à partir des eaux putrides provenant de la surface du sol qui entraînent avec elles les polluants vers la nappe phréatique. L'évaluation de l'état actuel de la pollution des eaux souterraines a montré une contamination progressive de la nappe par les nitrates du Sud vers le Nord en direction des nouveaux pôles d'habitation.

\section{REFERENCES}

Adjiri OA, Goné DL, Kouamé IK, Kamagaté B, Biémi J. 2008. Caractérisation de la pollution chimique et microbiologique de l'environnement de la décharge d'Akouédo, Abidjan, Côte d'Ivoire. In. J. Biol. Chem. Sci., 2(4): 401-410.

Aghui N, Biémi J. 1984. Bassin sédimentaire côtier, Géologie et Hydrogéologie des nappes de région d'Abidjan et risques de contamination. Annales Université Nationale, Côte d'Ivoire, série C, tome XX: 1-27.
AGHTM. 1992. Les Résidus Urbains Collectes et Nettoiement des Voies Publiques (Vol. 1). Edition Tec \& Doc; $357 \mathrm{p}$.

Aghzar N, Berdai H, Bellouti A, Soudi B. 2002. Pollution nitrique des eaux souterraines au Tadla (Maroc) Rev. Sci. Eau, 15(2): 459-492.

Ahoussi KE. 2008. Evaluation quantitative et qualitative des ressources en eau dans le Sud de la Côte d'Ivoire. Application de l'hydrochimie et des isotopes de l'environnement à l'étude des aquifères continus et discontinus de la région d'Abidjan-Agboville. Thèse, Université Cocody, Abidjan, Côte d'Ivoire, p. 270.

Baali F, Rouabhia A, Kherici N, Djabri L, Bouachaou L, Hani A. 2007. Qualité des eaux souterraines et risques de pollution en milieu semi-aride. Cas de la cuvette de Chéria (NE Algérien). Estudios Geológicos, 63(2): 127-133.

BCEOM. 1984. Conception générale des systèmes d'assainissement urbains dans le contexte africain, p. 166.

BNETD. 1996. Actualisation du schéma directeur d'Abidjan. Bilan diagnostic urbain. Etude n ${ }^{\circ}$. Phase 1, pp.1-153

BNETD. 2000. Actualisation Plan Directeur d'Assainissement et de Drainage de la ville d'Abidjan. Rapport préliminaire, p. 49.

Boukari M, Gaye CB, Faye A, Faye S. 1996. The impact of urban development on coastal aquifers near Cotonou, Benin. Journal of African Earth Sciences, 22(4): 403-408.

Broche J, Peschet JL. 1983. Enquête sur les pollutions actuelles et ponctuelles en Côte d'Ivoire. Réseau National d'observation de la qualité des eaux marines et lagunaires en Côte d'Ivoire. ORSTOM et Ministère de l'Environnement, Paris, p. 451.

Cabinet Roche. 1987. Plan directeur de récupération et d'élimination des déchets 
solides de la ville d'Abidjan. Rapport final, p.1-234.

Dongo K. 2001. Etude de l'évolution du système d'assainissement "Eaux Usées" de la ville d'Abidjan. Mém. DEA, Université Cocody, Abidjan, Côte d'Ivoire, p. 80.

Dongo K, Kouamé KF, Koné B, Biémi J, Tanner M, Cissé G. 2008. Analyse de la situation de l'environnement sanitaire des quartiers défavorisés dans le tissu urbain de Yopougon à Abidjan, Côte d'Ivoire. VertigO, 8(3): 1-11.

Faillat J-P, Squarcioni P, Hubert J-M. 1979. Utilisation de la prospection électrique pour l'étude de la géologie et de l'hydrogéologie du bassin sédimentaire côtier aux environs d'Abidjan (Forêt du Banco). Annales Université, Abidjan, série C (Sciences), tome XV.

Groupe Eoule. 2000. Proposition pour l'assainissement de la décharge d'Akouédo et de la production d'électricité à partir du biogaz. Rapport final, p.2-10.

Guiral D, Ferhi A. 1989. Caractérisation ionique et isotopique d'un système hydrologique tropical: la lagune Ebrié (Côte d'Ivoire). Oceanologica Acta, 12(1): 47-55.

Hani A, Djarbri L, Mania J. 1997. Etude des caractéristiques physico-chimiques du massif cristallophyllien de Séraïdi (nordest Algérien).Hard Rock Hydrosystems (Proceedings of Rabat Symposium S2 1997). IAHS Publ. N.241, pp. 41-59

INS 1998. Recensement général de la population et de l'habitat (RGPH). Données socio-démographiques et économiques des localités; résultats définitifs par localités, régions des lagunes, vol. III, tome 1, p. 43

Jourda JRP. 1987. Contribution à l'étude géologique et hydrogéologique du Grand Abidjan (Côte d'Ivoire). Thèse $3^{\text {è }}$ cycle, Université, Grenoble I, France, p. 299.
Koné M, Aka K. 1996. Les lagunes d'Abidjan asphyxiées par la pollution. Africa Geosciences Review, 3(3/4): 461-470.

Kouadio BH. 1997. Quelques aspects de la schématisation hydrogéologique : cas de la nappe d'Abidjan. Thèse de DEA, Université Cocody, Abidjan, Côte d'Ivoire, p.66.

Kouadio G, Dongui B, Trokourey A. 2000. Détermination de la pollution chimique des eaux de la zone de la décharge d'Akouédo-Abidjan, (Côte d'Ivoire). Rev. Sc. Tech., 1: 33-41.

Kouadio LP, Abdoulaye S, Jourda P, Loba M, Rambaud A. 1998. Conséquences de la pollution urbaine sur la distribution d'eau d'alimentation publique à Abidjan. Cahier de l'Association Scientifique Européenne pour l'Eau et la Santé, 3(1): 41-44.

Kouamé KJ. 2007. Contribution à la gestion intégrée des ressources en eaux (GIRE) du District d'Abidjan (Sud de la Côte d'Ivoire) : outils d'aide à la gestion pour la prévention et la protection des eaux souterraines contre la pollution. Thèse Université Cocody, Abidjan, Côte d'Ivoire, p. 227.

Mudry J, Blavoux B. 1986. Utilisation de l'analyse en composantes principales pour l'étude du fonctionnement hydrocynématique de trois aquifères karstiques du Sud-Est de la France. Hydrogéologie, (1): 53-59.

Oga YMS. 1998. Ressources en eaux souterraines dans la région du Grand Abidjan (Côte d'Ivoire): Approches hydrochimique et Isotopique. Thèse, Université Paris-Sud, France, p. 240.

Ouattara L. 2008. Impact des modes de gestion des déchets domestiques sur la qualité des eaux de la nappe d'Abidjan. Thèse de DEA, Université Cocody, Abidjan, Côte d'Ivoire, p. 80.

NEDECO. 1981. Assainissement et drainage de la ville d'Abidjan. Etude de facilité. 
Rapport d'actualisation du plan directeur, tome 2, p.1-57.

Sané Y. 1999. Une ville face à ses déchets ; une problématique géographique de la pollution à Abidjan (Côte d'Ivoire). Thèse de doctorat Université Laval, Québec, p. 290.

Sané Y. 2002. La gestion des déchets à Abidjan: un problème récurrent et apparemment sans solution. AJEAM/RAGÉE, 4(1): 13-22.

Travi Y, Mudry J. 1997. Méthode pour l'évaluation et la gestion du risque nitrate dans les aquifères de socle de la zone sahélienne d'Afrique de l'Ouest. Hydrogéologie, (1): 13-21.

Tandia AA, Gaye CB, Faye A. 1997. Origine des teneurs élevées en nitrates dans la nappe phréatique des sables quaternaires de la région de Dakar, Sénégal. Sécheresse, 8: 291-294.

Tandia AA, Diop ES, Gaye CB. 1999. Pollution par les nitrates des nappes phréatiques sous environnement semiurbain non assaini : exemple de la nappe de Yeumbeul, Sénégal. Journal African Earth Sciences, 22(4): 403-406. 